\title{
Predição de cobertura de sistemas em VHF e UHF em cenários com morfologias mistas
}

\author{
Guilherme M. O. Silva, José C. A. Santos e Maurício H. C. Dias
}

\begin{abstract}
Resumo-O presente trabalho descreve um algoritmo para predição de cobertura de sistemas VHF ou UHF de baixa potência, operando em cenários com diferentes morfologias. Ferramentas comerciais de predição costumam adotam métodos simplificados para resolver alguns problemas decorrentes do uso de modelos semi-empíricos com bases de dados geográficos. $O$ algoritmo desenvolvido aborda dois problemas em especial: a influência de terrenos mistos e a perda em trechos de vegetação densa. Seu teste foi na forma de rotinas em Matlab, comparando seus resultados aos obtidos usando o modelo ponto-área HataCOST-231. Os resultados foram coerentes com as características do terreno misto considerado na análise.
\end{abstract}

Palavras-Chave-Perda de percurso, VHF, UHF, predição de cobertura, terrenos mistos, modelo de Hata.

Abstract-This text describes an algorithm for coverage prediction of low power VHF or UHF radio-communication systems operating at mixed path scenarios. Commercial prediction tools either adopt simple methods for some issues expected in semi-empirical site-specific approaches or offer incomplete descriptive material, regarding their respective solutions. The proposed algorithm addresses two particular issues: the influence of mixed paths and the additional path loss due to dense vegetation. It has been tested as Matlab codes, comparing its results with the prediction carried out with the standard Hata-COST231 model. The results were coherent with the terrain and morphology information provided.

Index Terms-Path loss, VHF, UHF, coverage prediction, mixed paths, Hata model.

\section{INTRODUÇÃO}

A análise de cobertura é uma atividade fundamental no planejamento de sistemas de comunicações sem fio. Para sistemas de grande porte, como redes de telefonia celular, por exemplo, é usual o emprego de software especializado para predição das áreas de cobertura das estações que se planeja instalar numa determinada região de interesse. Tais programas manipulam bases de informações geográficas e sua função básica é o cálculo da perda média ou mediana de propagação a partir da aplicação de modelos próprios para a faixa de freqüência de operação do sistema, adaptados para aproveitar a disponibilidade de informações detalhadas sobre as áreas

Seção de Engenharia Elétrica, Instituto Militar de Engenharia, Rio de Janeiro, Brasil. E-mails: gmos1@hotmail.com; araujo@ime.eb.br; e mhcdias@ime.eb.br. analisadas. Esta estratégia de uso de modelos de predição com bases de dados geográficos, comum em ferramentas comerciais, é usualmente citada na literatura como abordagem site-specific [1]-[4].

Ferramentas site-specific lidam com a inexorável relação de compromisso entre tempo de processamento e acuidade da predição. Modelos teóricos, baseados em traçado de raios, têm maior capacidade de detalhar as variações do nível médio do sinal ao longo da área analisada, mas em compensação exigem grande esforço numérico. Para cada par de pontos tomado na área analisada (de transmissão e de recepção) há uma predição específica associada às trajetórias de propagação identificadas. Modelos semi-empíricos, por outro lado, demandam muito menos carga computacional, pois são expressões analíticas fechadas, próprias para predições pontoárea (PA), em que o aspecto mais relevante é o decaimento médio do sinal com a distância para uma dada frequência e um determinado tipo de ambiente. Uma solução intermediária, muito comum em ferramentas comerciais, é o uso de modelos semi-empíricos com ajustes adicionais ponto-a-ponto (PP) associados a aspectos extraídos das informações disponíveis sobre o terreno [1]-[4]. Um exemplo clássico desta abordagem é o uso do modelo de Hata [5] acrescido de um fator de perda adicional por difração no terreno.

$\mathrm{Na}$ estratégia de predição site-specific PA com ajustes PP, a aplicação de modelos semi-empíricos a bases de dados traz consigo várias dificuldades de implementação. A modelagem semi-empírica é baseada em medidas, e como tal, possui várias restrições de uso. Por sua vez, as bases de dados geográficas disponíveis possuem diferentes características e escopos de abrangência que também devem ser considerados na aplicação dos modelos.

Um problema importante, porém pouco comentado, de integração de modelos semi-empíricos a bases de dados é o tratamento de terrenos mistos. Todos os modelos semiempíricos se aplicam a um ou mais tipos de morfologia, como os modelos de Hata [5] e Lee [6], por exemplo. Entretanto, cada expressão desses modelos se aplica especificamente a um tipo de morfologia por vez. Não há expressões que se apliquem a enlaces passando por duas ou mais morfologias distintas. A solução mais simples para este problema é ignorar os efeitos de sucessivas transições de morfologia e em cada ponto de recepção calcular a perda respectiva assumindo os parâmetros da morfologia daquele ponto. Esta é a solução que a maioria das ferramentas comerciais adota provavelmente, mas tal informação nem sempre é claramente indicada. 
O presente trabalho descreve um algoritmo site-specific desenvolvido para predição de cobertura de sistemas operando em parte das bandas de VHF e UHF em cenários urbanos. A perda de propagação mediana é calculada com base no modelo de Hata-COST231 [1]-[2] e em ajustes PP ao longo de cada enlace. Bases de altimetria e morfologia são consideradas. Em particular, destacam-se como contribuições principais as soluções propostas para dois problemas de integração de modelos PA a bases de dados. Para o cálculo da perda em terrenos mistos, adotou-se o método de Millington [7]-[9]. E para a perda em trechos de bosques ou florestas densas, usou-se um modelo semi-empírico previsto na Recomendação UIT-R P.833 [10], baseado em medidas realizadas em uma área arborizada da cidade do Rio de Janeiro [11]-[12]. Resultados da implementação do algoritmo em Matlab são discutidos e comparados a abordagens mais simples, tomando como exemplo uma região de cerca de $11 \times 11 \mathrm{~km}^{2}$ da cidade de Campinas - SP, usando uma base de altimetria real e uma base de morfologia hipotética que se aproxima do cenário representado.

Este artigo foi estruturado como segue. A seção II descreve o cenário de aplicação do algoritmo, as bases de dados utilizadas e os modelos adotados. Resultados gerados pela implementação do algoritmo em Matlab no cenário exemplo adotado são apresentados e analisados na seção III. Por fim, a seção IV conclui o artigo.

\section{DESCRIÇÃO DO ALGORITMO}

\section{A. Cenários de Aplicação}

O algoritmo desenvolvido se aplica para determinação de áreas de cobertura de sistemas rádio de baixa potência operando em parte das faixas de VHF e UHF. Os cenários típicos são ambientes urbanos, em especial áreas que apresentem transições de morfologias. A faixa de operação considerada é entre $150 \mathrm{MHz}$ e $2 \mathrm{GHz}$, em função dos modelos de predição adotados.

A curvatura da terra foi desconsiderada no algoritmo, uma vez que para baixas potências e em ambientes urbanos, tipicamente a aproximação de terra plana se aplica.

\section{B. Bases de dados utilizadas}

Uma das premissas da solução site-specific adotada foi a disponibilidade de bases de dados de altimetria. As informações de alturas do terreno utilizadas pelo algoritmo desenvolvido foram obtidas do banco de dados gerado pela missão SRTM (Shuttle Radar Topographic Mission) [13][15]. Esta missão foi um projeto realizado em conjunto pela NASA (National Aeronautics and Space Administration) e NIMA (National Imaging and Mapping Agency), dos EUA, com participação das agências espaciais da Alemanha e da Itália, que em 2000 lançaram um equipamento SAR interferométrico a bordo do ônibus espacial Endeavour, operando nas bandas C e X. Ao longo de 11 dias, informações foram coletadas para gerar um mapa digital tridimensional de alta resolução de elevações de quase toda a superfície da Terra. A versão finalizada do mapa, contendo vários ajustes obtidos por pós-processamento das informações, foi disponibilizada em 2006. Há conjuntos de dados com grades de $30 \mathrm{~m}$ (SRTM 1) e $90 \mathrm{~m}$ (SRTM 3). Os dados de alturas obtidos possuem erro linear absoluto de $16 \mathrm{~m}$ e relativo de $10 \mathrm{~m}$. As bases SRTM 3 estão disponíveis livremente ao público em geral.

Para apoiar o presente trabalho, uma base SRTM 3 de parte da região Sudeste do Brasil foi copiada de [15], no formato geotiff. A partir da leitura da base disponível, é possível gerar gráficos de altimetria, como o mapa de superfícies da Fig. 1, que representa as alturas em uma área de $10,8 \times 10,8 \mathrm{~km}^{2}$ $(120 \times 120$ pixels $)$, correspondente a uma parte da cidade de Campinas - SP.

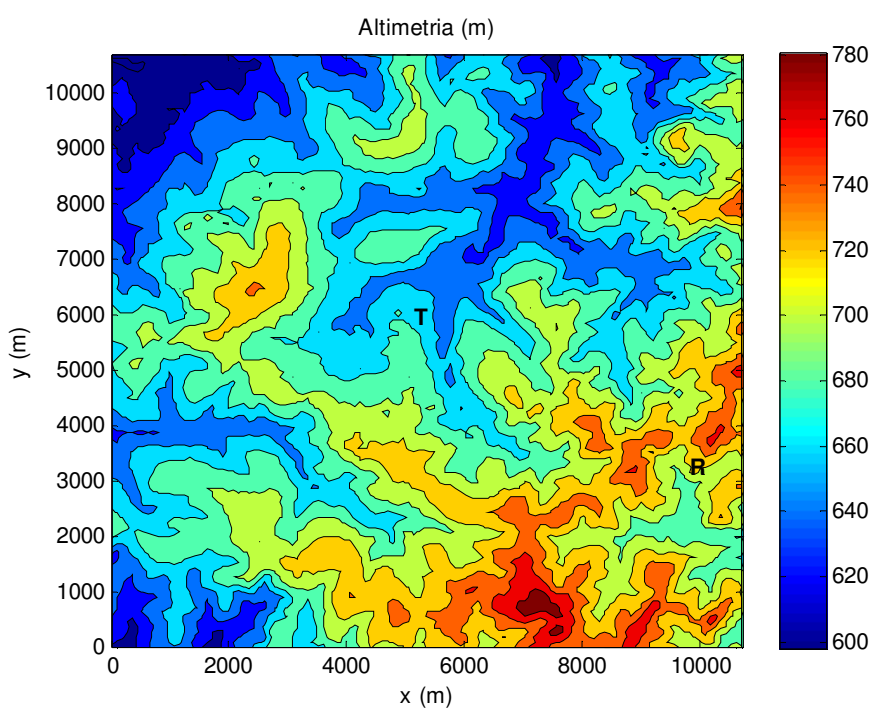

Fig. 1. Mapa de altimetria da região considerada.

A outra base de dados necessária ao algoritmo é a de morfologia. Para a mesma região da Fig. 1, considerou-se a distribuição hipotética de morfologias ilustrada na Fig. 2, que representa aproximadamente a realidade do local. O mapa de morfologias apresentado é um arquivo tiff, no qual cada cor representa um tipo de região distinta, de acordo com a associação indicada na Tabela I. Neste trabalho, sete diferentes morfologias foram assumidas.

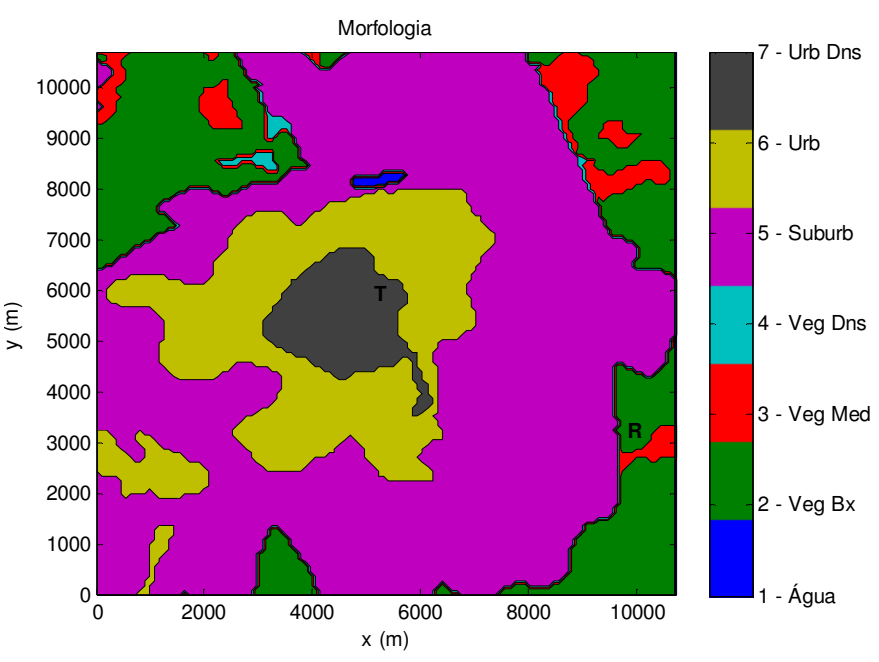

Fig. 2. Distribuição de morfologias na região considerada. 
TABELA I

MORFOLOGIAS ASSUMIDAS

\begin{tabular}{|c|c|}
\hline Número & Tipo de Região \\
\hline 1 & Água \\
2 & Vegetação Baixa \\
3 & Vegetação Média \\
4 & Vegetação Densa \\
5 & Suburbana \\
6 & Urbana \\
7 & Urbana Densa \\
\hline
\end{tabular}

\section{Modelos de predição de perda de propagação}

$\mathrm{O}$ algoritmo desenvolvido assume a estratégia site-specific ponto-área (PA) com ajustes ponto-a-ponto (PP) para o cálculo da perda mediana de percurso. $\mathrm{O}$ modelo PA base é o de Hata-COST 231 [1]-[4]. Os ajustes PP considerados foram: o tratamento de terrenos mistos (várias morfologias ao longo do mesmo enlace); a perda adicional por difração em ponto elevado do terreno; e a perda adicional por vegetação densa em áreas de bosques ou florestas.

Como dados de entrada para o algoritmo, além das bases de altimetria e morfologia já citadas, é necessário também indicar a posição da antena da estação transmissora na grade considerada (coordenadas horizontais e altura) e a potência na entrada da antena. Esta versão inicial do algoritmo assume antenas isotrópicas, pois $\mathrm{o}$ foco principal foram os mecanismos de propagação. Para inserção dos parâmetros de ganho das antenas transmissora e receptora, é preciso dispor de matrizes com os valores de ganho para um número razoável de azimutes e elevações $(360 \times 180$, por exemplo, assumindo resolução de $1^{\circ}$ ). A partir daí, poucas linhas de código são necessárias para calcular os ângulos de partida (antena transmissora) e chegada (antena receptora) em cada enlace, e buscar os valores correspondentes nas matrizes de ganhos. Os dois valores, em $\mathrm{dBi}$, são somados à potência recebida calculada assumindo antenas isotrópicas, como neste trabalho.

O modelo de Hata-COST 231 é bastante conhecido na literatura, e praticamente todas as ferramentas comerciais de predição de cobertura o incluem no rol de métodos disponíveis. Para freqüências entre $150 \mathrm{MHz}$ e $1,5 \mathrm{GHz}$, as expressões de Okumura-Hata são utilizadas. De $1,5 \mathrm{GHz}$ a 2,0 GHz, apenas a perda mediana urbana é dada por outra expressão, obtida a partir de campanhas de medidas complementares coordenadas pelos colaboradores da iniciativa européia COST-231. Os ganhos de altura e demais fatores de correção para outros ambientes do modelo de Hata se mantém.

O escopo de validade do modelo de Hata-COST 231 é para distâncias entre 1 e $20 \mathrm{~km}$, alturas efetivas de transmissão entre 30 e $200 \mathrm{~m}$, e alturas efetivas de recepção entre 1 e $10 \mathrm{~m}$. As alturas efetivas são calculadas tomando-se como referência a altura média do terreno entre o transmissor e o receptor, calculada entre $3 \mathrm{~km}$ e até $15 \mathrm{~km}$ a partir do transmissor. Entretanto, o algoritmo deve calcular a perda de percurso mesmo nos casos em que os limites de validade do modelo considerado são violados. Em função disso, no presente algoritmo, a média é calculada considerando toda a distância entre o transmissor e o receptor. Ainda, nos casos em que as alturas efetivas violam os limites de validade do modelo, os limiares mais próximos (inferior ou superior) são considerados para fins de cálculo dos respectivos ganhos de altura.

Para determinar a distribuição do sinal na área desejada, o algoritmo avalia a perda de propagação para todas as combinações de pares de posições transmissão-recepção possíveis na grade. Para cada enlace, o algoritmo extrai o perfil do terreno da base de altimetria, e o correspondente perfil de morfologias, da base morfológica. Se este perfil apresenta uma única morfologia, a perda mediana PA é calculada de acordo com a expressão apropriada do modelo de Hata-COST231. Vale destacar que os tipos de região de 5 a 7 da Tabela I são os mesmos previstos naquele modelo. Os tipos 1 a 4 da Tabela I, por sua vez, são tratados neste trabalho como área aberta, para fins de escolha da expressão de perda mediana PA.

Quando o perfil de morfologias é misto, o algoritmo usa o método de Millington para determinar a perda mediana PA correspondente. $\mathrm{O}$ método de Millington foi proposto originalmente como uma solução heurística para o cálculo da perda de percurso em terrenos mistos, para ondas terrestres em baixas frequências (até HF, inclusive) [7]. Medidas de campo do próprio autor e de outros indicaram a validade da solução. Posteriormente, o Setor de Radiocomunicações da UIT - União Internacional de Telecomunicações (UIT-R) incluiu o método em uma de suas recomendações [8]. A proposta de utilizar a mesma lógica para as frequências mais altas tratadas no presente trabalho também é heurística, inspirada em trabalho recente no qual o método de Millington foi avaliado para modelar a perda de propagação em transições de condição de visibilidade em cenários urbanos, para sistemas operando de 2 a $15 \mathrm{GHz}$ [16]-[17].

A perda mediana em um enlace misto $L_{M}(\mathrm{~dB})$ é dada por (1), que representa a média entre as perdas nos sentidos direto $\left(L_{D}\right)$ e reverso $\left(L_{R}\right)$, calculadas como indicado em (2) e (3), respectivamente. Os percursos cumulativos recursivos nos sentidos direto $\left(s_{k}\right)$ e reverso $\left(r_{k}\right)$, por sua vez, são definidos em (4) e (5), respectivamente. A Fig. 3 ilustra a composição destes percursos [9].

$$
\begin{gathered}
L_{M}=0,5\left(L_{D}+L_{R}\right) \\
L_{D}=\sum_{k=1}^{N} L_{k}\left(s_{k}\right)-\sum_{k=2}^{N} L_{k}\left(s_{k-1}\right) \\
L_{R}=\sum_{k=1}^{N} L_{k}\left(r_{k}\right)-\sum_{k=2}^{N} L_{k-1}\left(r_{k}\right) \\
s_{k}=\sum_{n=1}^{k} d_{n}=d_{1}+d_{2}+\cdots+d_{k} \\
r_{k}=\sum_{n=1}^{k} d_{N-n+1}=d_{N}+d_{N-1}+\cdots+d_{N-k}
\end{gathered}
$$




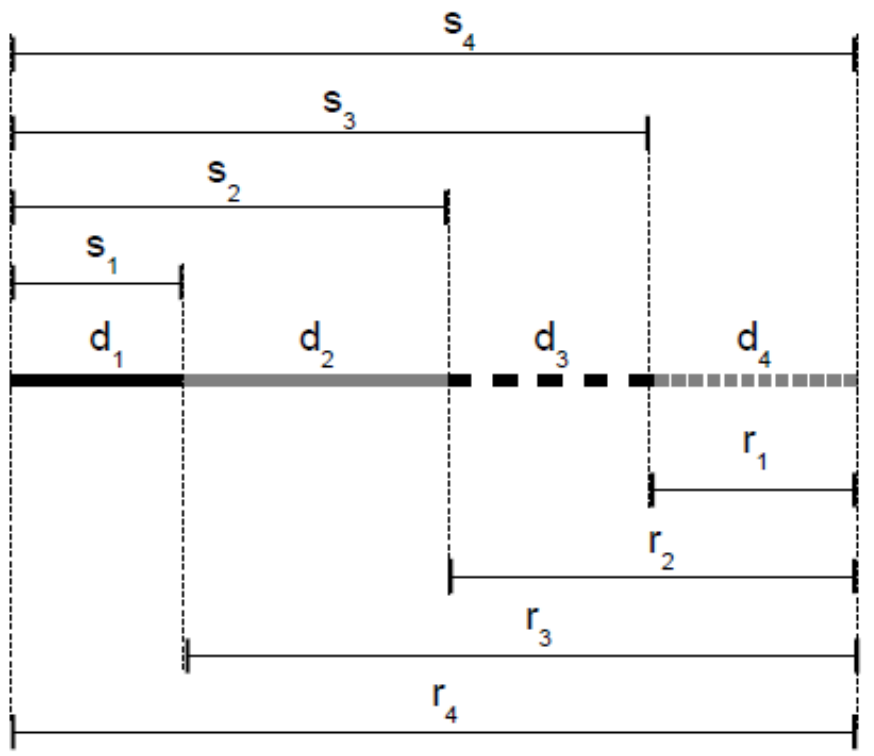

Fig. 3. Definição dos percursos $s_{k}$ e $r_{k}$ para as expressões recursivas de Millington em (3) e (4).

Outro ajuste decorrente da análise do perfil de morfologias é a soma da perda adicional decorrente da passagem do sinal por uma região do tipo 4 da Tabela I - vegetação densa. O modelo escolhido para este cálculo foi o proposto em [11][12], baseado em medidas, e que foi aceito como contribuição para a Recomendação P.833 do UIT-R [10]. A perda $L_{v}(\mathrm{~dB})$ é dada por:

$$
\begin{gathered}
L_{v}=A_{m}\left(1-e^{-\gamma \cdot d_{v} / A_{m}}\right) \\
A_{m}=0,18 f^{0,752} \\
\gamma=0,0063 f^{0,537}
\end{gathered}
$$

onde $d_{v}$ é a distância percorrida pelo sinal no trecho de vegetação densa, e $f$ é a frequência de operação $(\mathrm{MHz})$.

Por fim, a difração eventual por obstruções do elipsóide correspondente à primeira zona de Fresnel também é considerada. Mais especificamente, a análise é feita na interseção do elipsóide com o plano vertical que contém a antena de transmissão e o ponto de recepção. Neste trabalho, a perda adicional por difração é calculada pelo modelo mais simples disponível em [18], que considera apenas a contribuição do obstáculo principal ao longo do perfil, modelando-o como um "gume-de-faca". A perda correspondente $L_{d}(\mathrm{~dB})$ é dada por uma integral de Fresnel, que em [18] é expressa pela aproximação:

$$
L_{d}=6,9+20 \log \left(\sqrt{(v-0,1)^{2}+1}+v-0,1\right)
$$

onde $v=\sqrt{2} \cdot h / r_{f 1}$ é o parâmetro de Fresnel e $r_{f 1}=\sqrt{\lambda d_{1} d_{2} /\left(d_{1}+d_{2}\right)}$ é o raio da $1^{\mathrm{a}}$ zona de Fresnel. As distâncias envolvidas $d_{1}$ e $d_{2}$ são definidas a partir da geometria base ilustrada na Fig. 4 , e $\lambda$ é o comprimento de onda do sinal difratado. A perda $L_{d}$ só deve ser calculada para $v \geq-0,7$, caso contrário, $L_{d}=0$. Vale destacar que, no algoritmo desenvolvido, o paramêtro de Fresnel é calculado para todos os pontos do perfil, de modo a escolher como obstáculo principal aquele que leve ao maior valor de $v$.

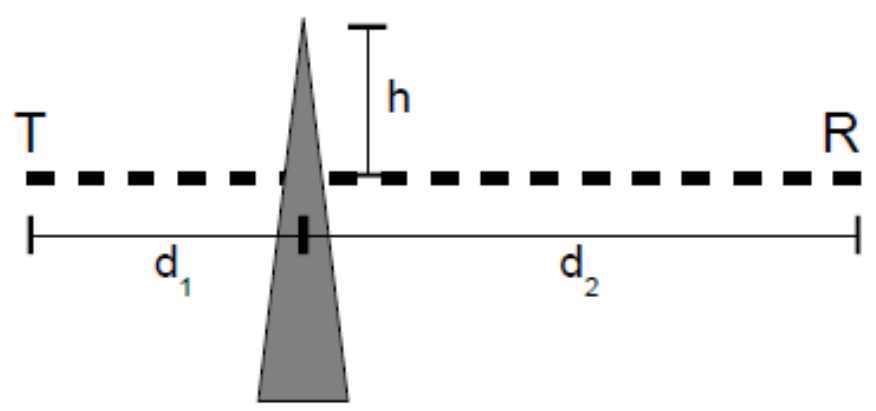

Fig. 4. Geometria base para o cálculo da perda adicional por difração.

\section{ANÁLISE DOS RESULTADOS}

O algoritmo foi implementado e testado na forma de rotinas para Matlab. Alguns conjuntos de enlaces foram selecionados para observar a influência dos parâmetros de ajustes PP considerados no algoritmo desenvolvido, de modo a avaliar sua coerência e correção. Para tal, junto com os resultados do algoritmo, foram calculados os valores de perda de percurso assumindo apenas o modelo de Hata-COST231 para a morfologia predominante de cada enlace, sem ajustes adicionais.

A Fig. 5 apresenta a primeira comparação de resultados do algoritmo com o modelo PA base adotado. Foram calculadas as perdas de percurso ao longo do perfil entre o ponto $\mathrm{T}$ de coordenadas $x_{T}=5,22 \mathrm{~km}$ e $y_{T}=5,85 \mathrm{~km}$ e o ponto $\mathrm{R}$ de coordenadas $x_{R}=9,90 \mathrm{~km}$ e $y_{R}=3,15 \mathrm{~km}$, nas grades ilustradas nas Figs. 1 e 2. Assumiu-se que a potência de transmissão $P_{T}$ era de $50 \mathrm{dBm}$, que a altura da antena de transmissão $h_{T}$ era de $30 \mathrm{~m}$, que a altura da antena de recepção $h_{R}$ era de $1,5 \mathrm{~m}$, e que a frequência $f$ era de 1,85 GHz. Percebe-se na Fig. 5 que o perfil considerado apresenta diversas transições de morfologia e que as variações de altura do terreno causam difração em vários pontos de observação. Logo no trecho inicial $(d \approx 700 \mathrm{~m})$, em que não há difração, já é possível perceber o efeito da transição de morfologia (urbana densa para urbana), computada pelo algoritmo usando a metodologia de Millington. Em pontos mais afastados, a difração e a influência acumulada das diversas morfologias ficam mais evidentes. Por exemplo, na transição do ambiente urbano (6) para suburbano (5) em $d \approx 1,9 \mathrm{~km}$, percebe-se um leve acréscimo no sinal recebido. Entretanto, nos trechos seguintes, a intensidade do sinal cai mais rapidamente que a predita pelo modelo PA na morfologia predominante, devido à difração. Os "degraus" comuns em ambas as curvas de predição $(d \approx 5 \mathrm{~km})$ estão associados aos efeitos das variações do terreno assimilados pelos ganhos de altura do modelo PA base. 

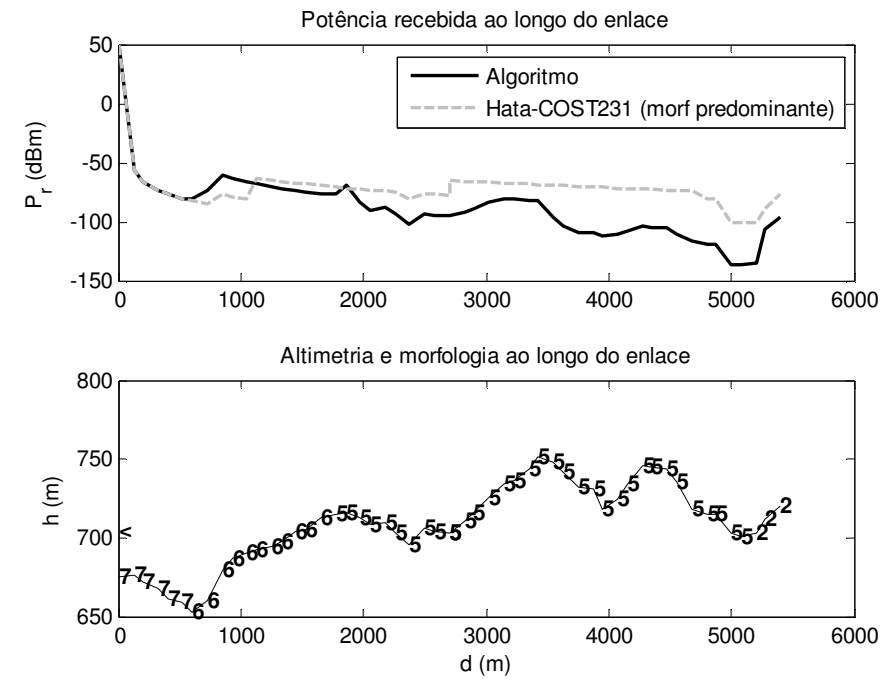

Fig. 5. Potência recebida ao longo de um perfil com diferentes morfologias.

Para observar o efeito específico da perda adicional por vegetação, escolheu-se o perfil entre os pontos $(2,25 \mathrm{~km}$, $8,82 \mathrm{~km})$ e $(3,96 \mathrm{~km}, 8,10 \mathrm{~km})$. A Fig. 6 apresenta os resultados obtidos para este perfil, assumindo os mesmos valores de $P_{T}, h_{T}, h_{R}$ e $f$ da comparação anterior. Observa-se na curva de altimetria e morfologia do terreno que a distância máxima é relativamente curta $(<2 \mathrm{~km})$, com grande proporção de vegetação densa. Na maior parte do trecho, até 1,4 km, a expressão PA que se aplica é a mesma (para área aberta). A diferença observada diz respeito principalmente à perda por vegetação ao longo do trecho (há perda por difração, baixa, em dois pontos). A partir de $1,4 \mathrm{~km}$, a transição para área suburbana aumenta ainda mais a diferença para o modelo PA de comparação.
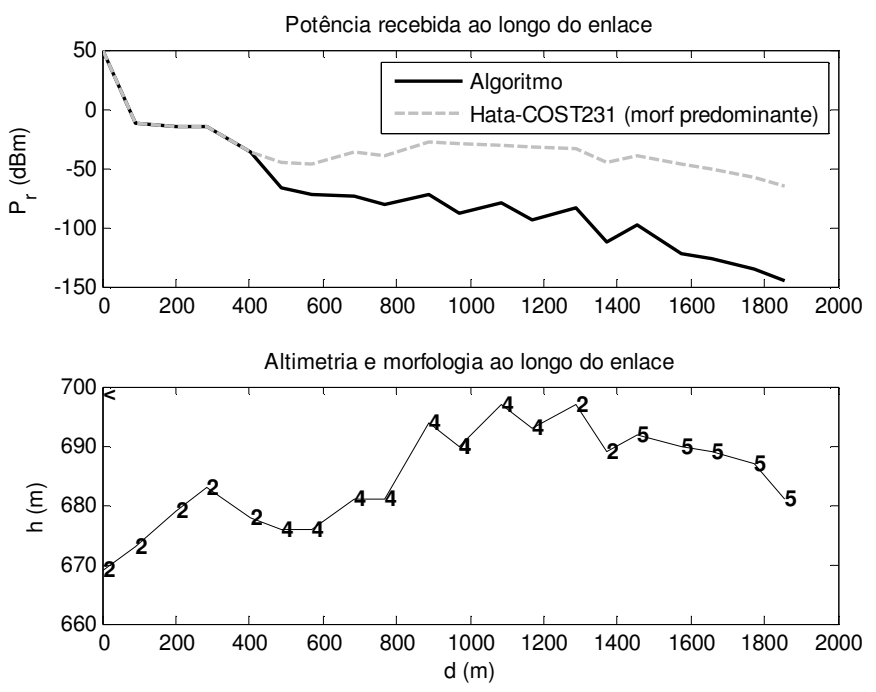

Fig. 6. Potência recebida ao longo de um perfil que atravessa trecho de vegetação densa.

Mantendo os mesmos valores de $P_{T}, h_{T}, h_{R}$ e $f$ das comparações anteriores, o algoritmo foi executado para calcular a perda de percurso em todos os pontos da área considerada neste trabalho, exceto o de transmissão, em $x_{T}=0,90 \mathrm{~km}$ e $y_{T}=9,90 \mathrm{~km}$. A Fig. 7 apresenta o resultado correspondente, indicando a distribuição da potência mediana recebida em toda a área. Comparando a Fig. 7 com as Figs. 1 e 2, é possível identificar a influência da difração e da perda por vegetação em vários pontos da região. Por exemplo, os "feixes" de valores entre -120 e $-140 \mathrm{dBm}$ observados a partir da posição do transmissor (T) na direção diagonal inferior do mapa $(x=10,5 \mathrm{~km}$ e $y<9 \mathrm{~km})$ são devidos à passagem do sinal pelo trecho de vegetação densa (em torno de $x=3 \mathrm{~km} \mathrm{e}$ $y=9 \mathrm{~km}$ na Fig. 2). A difração, por sua vez, pode ser observada pela redução de sinal na região em torno de $x<3 \mathrm{~km}$ e $y=4 \mathrm{~km}$, que corresponde a um vale (vide Fig. 1).

A influência das transições de morfologia, entretanto, não é tão evidente neste tipo de representação, particularmente no presente cenário, em que as variações de altura do relevo são significativas. Um exemplo desta dificuldade em discriminar causa e efeito é o trecho em torno de $x=8,5 \mathrm{~km}$ e $y>8,5 \mathrm{~km}$, onde se observa um claro reforço no sinal recebido. Esta região é um pouco mais alta que os vales ao seu redor, ao mesmo tempo que reresenta uma transição entre ambiente suburbano (5) e aberto ( 2 e 3 ). Com isso, o reforço de sinal pode ter sido consequência tanto de uma menor perda por difração, quanto da transição para uma morfologia menos atenuante, ou mesmo de ambos os fatores.

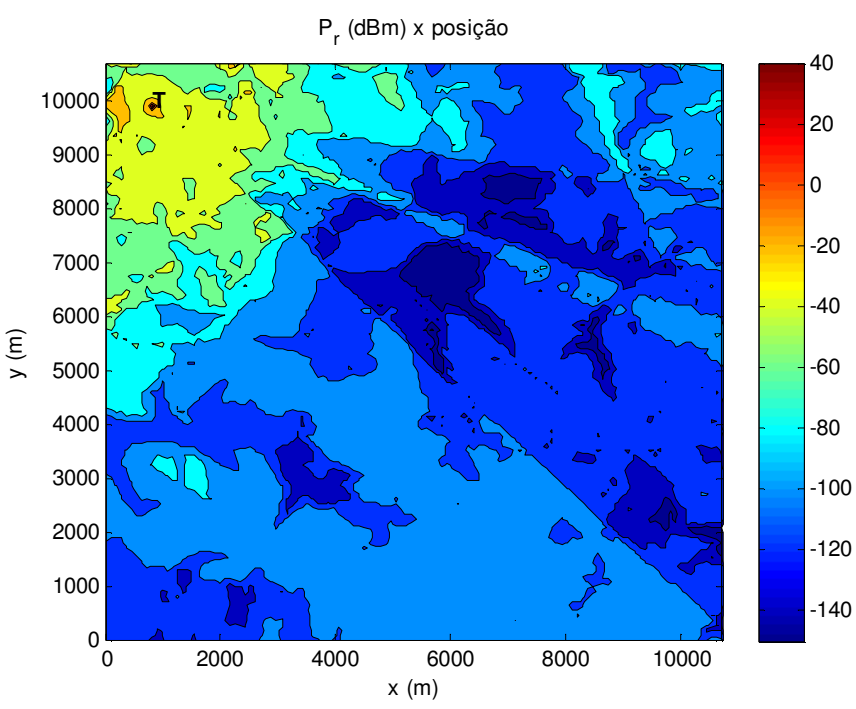

Fig. 7. Distribuição de potência recebida na região do exemplo considerado.

\section{CONCLUSÃO}

Este trabalho descreveu um algoritmo para predição de cobertura de sistemas de rádio-comunicação de baixa potência operando em parte das bandas de VHF e UHF em cenários com diferentes morfologias. A falta de informações detalhadas sobre as soluções adotadas para certos problemas esperados em abordagens site-specific semi-empíricas, seja na literatura, seja no material descritivo de ferramentas comerciais, motivou o desenvolvimento de um algoritmo com propostas para duas questões, em especial: influência de terrenos mistos e perda em trechos de vegetação densa.

$\mathrm{O}$ algoritmo foi desenvolvido com base na abordagem PA semi-empírica, com ajustes PP. O modelo PA base adotado foi o de Hata-COST231. A influência de morfologias mistas em um mesmo percurso foi avaliada a partir do uso de uma 
solução heurística: o método de Millington. A perda adicional por vegetação densa, por sua vez, foi modelada no algoritmo de acordo com formulação empírica indicada na Recomendação P.833 do UIT-R. A difração no terreno também foi considerada no algoritmo, tomando a difração de Fresnel no obstáculo principal ao longo de cada enlace.

Rotinas em Matlab foram implementadas para testar o algoritmo. Os resultados obtidos foram comparados com o modelo PA base em várias situações, e observou-se coerência dos valores de perda de percurso calculados com os dados correspondentes de altimetria e morfologia considerados. Validações adicionais ainda são necessárias, entretanto, em especial com base em medidas, ou com resultados de ferramentas comerciais.

\section{REFERÊNCIAS}

[1] T. S. Rappaport, Wireless Communications: Principles and Practice, $2^{\text {nd }}$ Ed, Prentice-Hall, 2001.

[2] J. D. Parsons, The Mobile Radio Propagation Channel, $2^{\text {nd }}$ Ed, Wiley, 2000.

[3] H. L. Bertoni, Radio Propagation for Modern Wireless Systems, Prentice-Hall, 2000.

[4] T. K. Sarkar, Z. Ji, K. Kim, A. Modouri, M. S.-Palma, "A Survey of Various Propagation Models for Mobile Communication", IEEE Antennas and Propagation Magazine, vol. 45, no. 3, pp. 51-82, Jun 2003.

[5] M. Hata, "Empirical formula for propagation loss in land mobile radio services", IEEE Transactions on Vehicular Technology, vol. 29, no. 3, pp. 317-325, Ago 1980.

[6] W. C. Y. Lee, Mobile Communications Engineering: Theory and Applications, $2^{\text {nd }}$ Ed, McGraw-Hill, 1998.

[7] G. Millington, "Groundwave Propagation over an Inhomogeneous Smooth Earth", IEE Proceedings, vol. 96, p. 53, Mar 1949.

[8] Groundwave Propagation Curves for Frequencies Between $10 \mathrm{kHz}$ and $30 \mathrm{MHz}$, Rec. UIT-R P.368-7, 1992.

[9] L. Sevgi, "A Mixed-Path Groundwave Field-Strength Prediction Virtual Tool for Digital Radio Broadcast Systems in Medium and Short Wave Bands", IEEE Antennas and Propagation Magazine, vol. 48, no. 4, pp. 19-27, Ago 2006.

[10] Attenuation in Vegetation, Rec. UIT-R P.833-6, 2007.

[11] M. H. C. Dias, Análise Crítica da Propagação em Microcélulas, dissertação de Mestrado, Instituto Militar de Engenharia, Rio de Janeiro - RJ, Jan 1998.

[12] M. H. C. Dias, M. S. Assis, "Considerações sobre a Propagação em Microcélulas em Áreas Urbanas Densas", in VIII Simpósio Brasileiro de Microondas e Optoeletrônica, Joinville - SC, vol. 1, pp. 266-270, Jul 1998.

[13] T. G. Farr, P. A. Rosen, E. Caro, R. Crippen, R. Duren, S. Hensley, M. Kobrick, M. Paller, E. Rodriguez, L. Roth, D. Seal, S. Shaffer, J. Shimada, J. Umland, M. Werner, M. Oskin, D. Burbank, D. 1sdorf, "The Shuttle Radar Topography Mission", Reviews of Geophysics, vol. 45, no. RG2004, Mai 2007.

[14] P. R. A. Santos, C. Gaboardi, L. C. Oliveira, "Avaliação da precisão vertical dos modelos SRTM para a Amazônia", Revista Brasileira de Cartografia, vol. 58, no. 1, pp. 101-107, Abr 2006.

[15] Sítio da missão SRTM, em http://www2.jpl.nasa.gov/srtm, acessado em 24 Abr 2009.

[16] R. M. L. Silva, Características da propagação ponto-área na faixa de 2 a $15 \mathrm{GHz}$ com aplicações em comunicações móveis, dissertação de mestrado, Instituto Militar de Engenharia, Rio de Janeiro - RJ, Mai 2004.

[17] R. M. L. Silva, M. S. Assis, "Previsão da atenuação de propagação em sistemas ponto-área na faixa entre 2 a 15 GHz", in MOMAG 2004 Simpósio Brasileiro de Microondas e Eletromagnetismo, São Paulo, Ago 2004, pp 1-6.

[18] Propagation by Diffraction, Rec. UIT-R P.526-10, 2007. 